\title{
REDUCING THE MUSCLE CHAIN HYPERTONIA THROUGH MYOFASCIAL TECHNIQUES IN ATHLETES
}

\author{
Bogdan ANTOHE ${ }^{*}$, Gloria RAȚ $\breve{A}^{2}$, Marinela RAȚA $\breve{A}^{2}$ \\ ${ }^{1}$ National University of Physical Education and Sport, Faculty of Physical Education and Sport, Bucharest, \\ Romania \\ 2 “Vasile Alecsandri” University, Faculty of Movement, Sport and Health Sciences, Bacău, Romania \\ *Corresponding author: antohe_bogdan@yahoo.com
}

https://doi.org/10.35189/dpeskj.2020.59.2.9

\begin{abstract}
It has been talked about the existence of muscle chains since the early 1960s, but they were described only in the early 2000s by Myers. From a structural and functional point of view, the muscle chains facilitate the integration of the human body movements into a global concept based on the human biomechanical principles. The concept starts from the idea that the muscles of the human body do not function in isolation, but they are considered part of a tensegrity system, being bound and oriented through the fascia. This paper aims to evaluate and treat muscle chains that have scientifically proven anatomical and functional connections in 4 professional athletes ( 2 boys and 2 girls, with an average age of 21 years). Their muscle activity was recorded by using surface electromyography (Biopac MP36). The values obtained were introduced into tables and analysed according to the theory of muscle chains in order to design a treatment scheme based on myofascial release techniques. The results of the research demonstrate the existence of functional bonds between the evaluated muscle chains and the effectiveness of myofascial release techniques in their treatment.
\end{abstract}

Keywords: muscle chains, athletics, myofascial.

\section{Introduction}

The history of muscle chains begins in 1950, when Dr. Kabbat laid the foundation for the neuro-proprioceptive facilitation techniques. Shortly afterwards, Struyff described the muscle chains in relation to the psychological component of the human posture. In the 1980s, Busquets and Chauffour followed, the list ending with Myers, whose book, Anatomy Trains, was re-printed for the third time in 2012 (Richter \& Hebgen 2009).

From a structural and functional point of view, the muscle chains facilitate the integration of the human body movements into a global concept based on the human biomechanical principles (Myers, 2009). The concept starts from the idea that the muscles of the human body do not function in isolation, but they are considered part of a tensegrity system, being bound and oriented through the fascia (Budiman, 2009). The anatomical connections between muscle chains are made through the connective tissue, especially through the superficial and deep fascia (Lee et al., 2011). A fascia is a connective tissue organized in the form of a threedimensional matrix, which surrounds, supports, suspends, protects, binds and divides the muscular, skeletal and visceral components of the body (Tozzi, 2012).

Epimysium, perimysium and endomysium are extensions of the deep fascia and have an anatomical continuity up to the level of superficial fascia (Stecco \& Day, 2010). They may extend beyond the limits imposed by the muscle fibre to form tendons and aponeuroses that bind muscle to muscle or muscle to periosteum (Manheim, 2001). The link between a muscle and fascia is called a 'myofascial unit' (Stecco, 2004). Through the myofascial unit, the 
muscle strength is not transmitted directly from the tendon to the bone, much of it being transmitted to the adjacent structures (synergistic muscles) and, surprisingly, to the antagonistic muscles (Huijing, 2009).

In time, over 20 muscle chains have been described, some of which are based on anatomical connections, others are based on the authors' logical deductions (Dischiavi et al., 2018). In the present paper, we used 4 muscle chains (the anterior and posterior spiral chain, the anterior and posterior superficial chain) described by Myers, whose existence has been scientifically demonstrated (Krause et al., 2016).

According to the Educational Council of Osteopathic Principles, myofascial release techniques are a component of manual therapy and have the role of diagnosing and treating various disorders of the musculoskeletal system (Ajimsha et al., 2015). They require the application of moderate but constant pressure on the connective tissue to reduce muscle tension and mobility constraints. Myofascial release represents a "philosophy", a clinical reasoning and not a summation of techniques that apply according to a pre-established protocol. The essence of myofascial release techniques consists in the feedback provided by the subject to determine the strength, direction and duration of application. The technique ends when we have a feeling of heat (as a result of reflex vasodilation) and a decrease in tension under our hands. The physiological explanation of the myofascial release is based on the viscoelastic properties of the connective tissue that reacts to a mechanical stimulus by changing the consistency of the fundamental substance and by breaking the transverse bridges between the connective and muscle tissue layers (Maganaris \& Paul, 2000).

There is some research that has shown that muscle chains have the capacity to transfer the contraction force through the connective tissue (Huijing, 2012). The strongest evidence relates to the plantar fascia, triceps surae muscles, hamstring muscles and sacrotuberous ligaments (Krause et al., 2016).

Given the evidence that muscle chains exist and have the capacity to transmit contraction forces, we can assume that, when the anatomical or functional integrity of a muscle chain is impaired, the repercussions occur not only locally but also across the entire muscle chain (do Rosário et al., 2012). Knowing all these aspects, we start from the premise that the development of the clinical judgment, according to the theory of muscle chains, offers a new perspective to the physical therapeutic practice, based on entirety, which can bring many benefits to patients.

\section{Methodology}

The aim of this paper was to identify the hypotonic muscle chains based on visual, palpation and electromyographic examinations, and in the light of the results obtained, we have developed a personalised, individualised protocol according to the principles of muscle chains.

The research issue is the following: we assume that, by applying a therapeutic protocol based on myofascial release techniques, muscle rebalancing will be achieved and at the same time a relaxation of the entire muscle chain with which it is connected. 
The research methods used were: the theoretical documentation method, the measurement and evaluation method, the observation method, the graphical method, the data recording method.

\section{Participants}

Four athlete subjects ( 2 boys and 2 girls), with an average age of 21 years, took part in the study (Table 1).

Table 1. Subjects involved in the research

\begin{tabular}{|c|c|c|c|c|c|c|}
\hline No. & Name & Gender & Age & Affected muscle chain & Competition period & Existent injuries \\
\hline 1 & P.A. & $\mathrm{F}$ & 23 & $\begin{array}{l}\text { P.R. spiral chain } \\
\text { A.L. spiral chain }\end{array}$ & Training & $\begin{array}{l}\text { Post-operation } \\
\text { Haglund disease }\end{array}$ \\
\hline 2 & P.G. & M & 21 & $\begin{array}{l}\text { P. superficial chain } \\
\text { P.R. spiral chain } \\
\text { P.L. spiral chain } \\
\text { A. Sunerficial chain }\end{array}$ & Training & $\begin{array}{c}\text { R. foot } \\
\text { Iliotibial friction } \\
\text { syndrome L. Foot } \\
\text { Flat Foot }\end{array}$ \\
\hline 3 & S.O. & F & 20 & $\begin{array}{l}\text { A. superficial chain } \\
\text { P.R. spiral chain } \\
\text { P.L. spiral chain }\end{array}$ & Training & $\begin{array}{l}\text { Suspect of R. femoral } \\
\text { osteosarcoma* }\end{array}$ \\
\hline 4 & U.S. & M & 20 & $\begin{array}{l}\text { A. superficial chain } \\
\text { A. L. spiral chain } \\
\text { P. L. spiral chain } \\
\text { A. R. chain }\end{array}$ & Training & - \\
\hline
\end{tabular}

\section{Procedure}

The research was carried out at the Athletics Hall in Bacău. The treatments were applied for 8 weeks, 2 times a week, with an average duration of 40 minutes. The research was conducted between March 25 and May 31, 2019. For evaluation, we used visual examination, palpation and surface electromyography (Biopac MP36). The objectives of the recovery programme were: to reduce muscle tone, especially in the areas of maximum tension; to increase muscle elasticity and joint mobility; to combat connective tissue adhesions; to facilitate muscle synergy and eliminate wrong movement patterns; to prevent injuries.

Content of the therapeutic programme:

The therapeutic programme consisted in applying myofascial release techniques. These are performed by placing a medium pressure on the skin, which is intended to penetrate each myofascial layer and induce relaxation. The application speed was related to the tension in each muscle area. The literature recommends "feeling" the tissue and guiding the pressure to the direction dictated by it. The technique can be improved by helping the patients' inhaling and exhaling or active movements, the process being called 'active myofascial release'.

The protocol used for the subject no. 1, P.A., consisted in the application of myofascial release techniques, especially on the injured right foot. Since the subject had given up for 1 week on the plaster cast, the first 4 treatments were local, having the role of smoothing the scar and increasing joint mobility. In the following weeks, we focused our attention on the lower half of the posterior left chain (triceps surae muscles, hamstrings muscles and gluteal muscles). The last treatment was applied before the final evaluation and aimed at rebalancing 
the subject at the level of all muscle chains. In the case of subject no. 2, P.G., the intervention was based on the concept of muscle chains. In the initial evaluation, the athlete had a right flat foot and hypertonia of the posterior right spiral chain. The treatment aimed at relaxing the muscles responsible for the flat foot (triceps surae muscle, tibialis anterior) and releasing the posterior right spiral chain (plantar fascia, left triceps surae muscles, hamstring muscles, gluteal muscles and all the right paravertebral mass). After the 4th treatment, the pain diminished, and the subject was able to resume his sports activity.

Due to the pre-existing injury, the treatment applied to subject no. 3, S.O., focused on eliminating muscle tension from the left leg (quadriceps muscle, tibialis anterior muscles, hamstring muscles and triceps surae muscle). After the athlete resumed her sports activity, we intervened on the anterior chain (pectoralis major muscles, rectus abdominis muscles, quadriceps muscles and tibialis anterior muscles). The last 4 treatments were applied only on the left hamstring muscles, the patient being in the recovery period after a muscle strain.

For the subject no. 4, S.U., the intervention focused on the upper half of the anterior right chain (pectoralis major and rectus abdominis) and the lower half of the posterior right chain (plantar fascia, triceps surae muscle and hamstring muscles). We applied this reasoning because the subject presented a kyphotic attitude generated by the hypertonia of the anatomical flexion chain. After each treatment, the athlete reported increased mobility of the trunk and a release of muscle tension, especially in the calves.

The effects of myofascial release techniques were improved by applying a personalised stretching programme before each workout, weekly modified depending on the treatment scheme applied. All muscles that had the myofascial release techniques applied on were subjected to stretching 3 times a day for 2 minutes.

\section{Results}

The results obtained by the athletes will be presented in the following tables and figures.

Table 2. Muscle contraction values $(\mathrm{mV})$ for the subject P.A.

\begin{tabular}{lcccc}
\hline \multicolumn{1}{c}{ Measured muscle } & MIVR $(\mathrm{mV})$ & FMVR $(\mathrm{mV})$ & IVMC $(\mathrm{mV})$ & FVMC $(\mathrm{mV})$ \\
\hline R. pectoralis major & 0.078 & 0253 & 1.000 & 0.686 \\
L. pectoralis major & 0.097 & 0.116 & 1.378 & 0.916 \\
R. rectus abdominis & 0.192 & 0.064 & 2.237 & 0.870 \\
L. rectus abdominis & 0.177 & 0.049 & 1.362 & 0.882 \\
R. quadriceps & 0.023 & 0.040 & 3.361 & 4.026 \\
L. quadriceps & 0.031 & 0.061 & 2.259 & 3.334 \\
R. tibialis anterior & 0.120 & 0.014 & 1.169 & 1.665 \\
L. tibialis anterior & 0.033 & 0.090 & 3.713 & 2.067 \\
R. rhomboid major & 0.108 & 0.130 & 3.865 & 1.488 \\
L. rhomboid major & 0.352 & 0.106 & 2.615 & 2.393 \\
R. paravertebral & 0.108 & 0.094 & 2.122 & 2.877 \\
L. paravertebral & 0.029 & 0.068 & 1.970 & 2.854 \\
R. hamstrings & 0.224 & 0.101 & 1.880 & 1.288 \\
L. hamstrings & 0.243 & 0.068 & 2.371 & 2.508 \\
R. triceps surae & 0.101 & 0.073 & 0.568 & 2.637 \\
L. triceps surae & 0.034 & 0.118 & 3.027 & 1.508 \\
\hline Legend: MIVR $=$ initial maximum value at rest, FMVR $=$ final maximum value at rest, IVMC $=$ initial value at maximum contraction, \\
FVMC $=$ final value at maximum contraction, $R=$ right, $L=$ left, mV $=$ millivolt & &
\end{tabular}




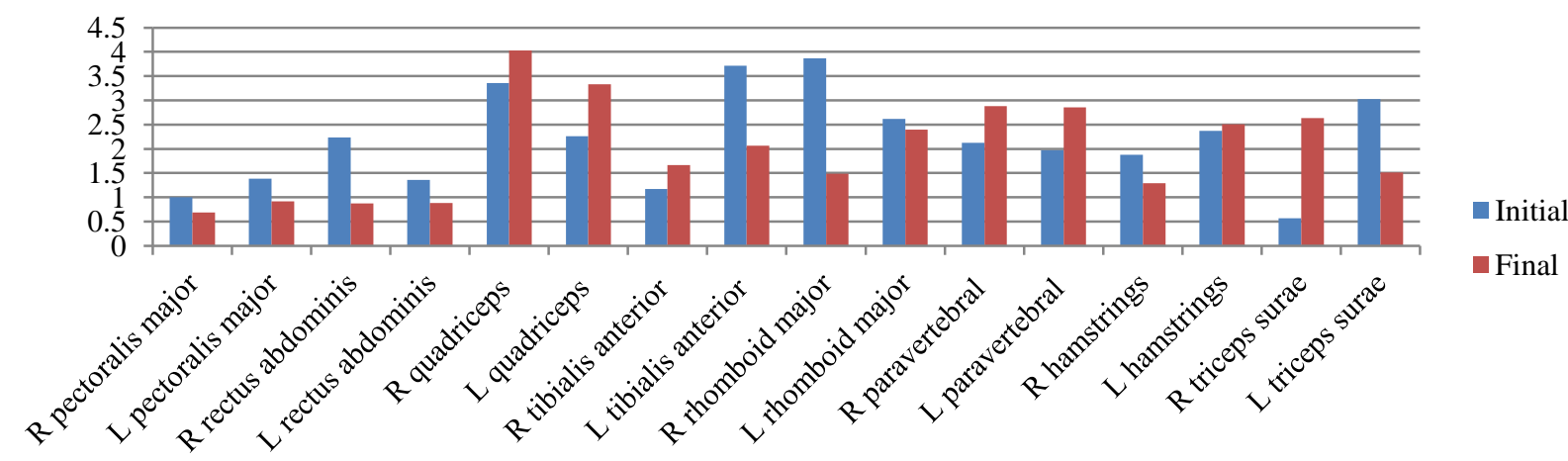

Figure 1. Dynamics of the muscle tone evolution during maximum contraction for the subject P.A.

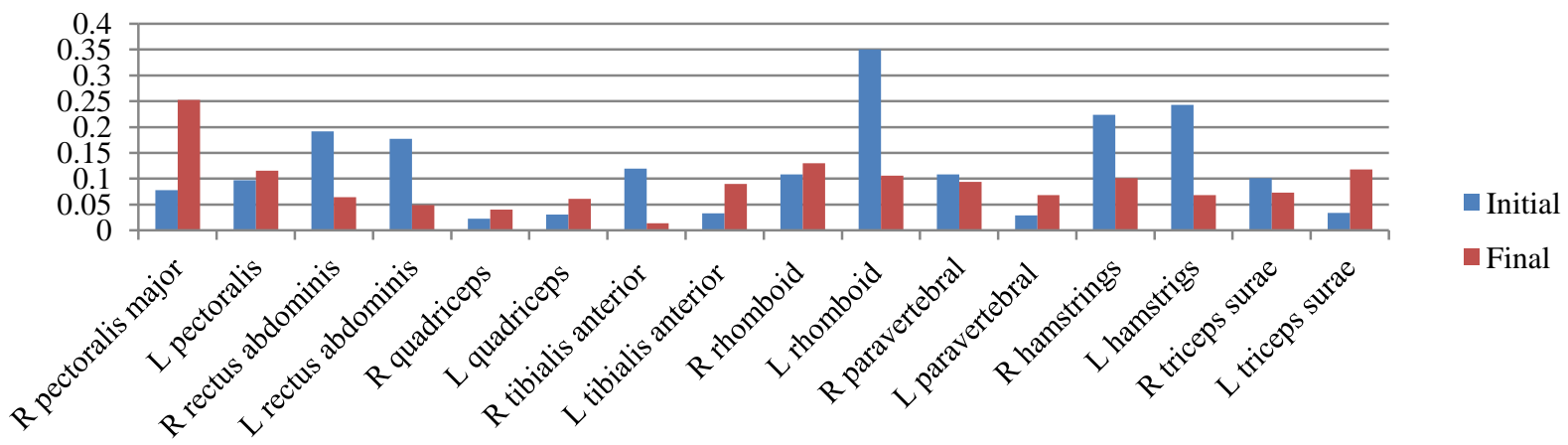

Figure 2. Dynamics of the muscle tone evolution at rest for the subject P.A.

Figure 1 highlights the evolution of muscle tone values during isometric contraction. The most important changes occur in the right triceps surae muscle, whose values have increased 4 times $(0.568 \mathrm{mV}$ initially $-2.637 \mathrm{mV}$ finally). In parallel, a half-decrease in the left triceps surae muscle (3.027 mV initially $-1.508 \mathrm{mV}$ finally) and left tibialis anterior muscle (3.713 $\mathrm{mV}$ initially $-2.067 \mathrm{mV}$ finally) can be observed. This reversal of values is normal because the athlete had resumed her sports activity 1 week before the evaluation, therefore her muscle tone was diminished in the right lower limb.

Regarding the muscle chain activity, we found that there was an increase of $0.220 \mathrm{mV}$ in functional extension chain (initially $2.473 \mathrm{mV}$ - finally $2.473 \mathrm{mV}$ ) at the final evaluation and a decrease of $0.350 \mathrm{mV}$ in functional flexion chain (initially $1.718 \mathrm{mV}$ - finally $1.358 \mathrm{mV}$ ) at the final evaluation. Another change is encountered in the anatomical extension chain that has decreased its activity by $0.110 \mathrm{mV}$ (initially $2.302 \mathrm{mV}$ - finally $2.194 \mathrm{mV}$ ). These changes occur because, when resuming the sports activity, the muscle groups of the extension chain are the ones that contribute the most to the biomechanics of running.

Another important aspect is the reversal of the contraction capacity of the posterior spiral chains. The left posterior chain $(1.796 \mathrm{mV}$ initially $-2.006 \mathrm{mV}$ finally), specific to the righthanded, finally became more active compared to the right posterior chain ( $2.808 \mathrm{mV}$ initially - $2.061 \mathrm{mV}$ finally). 
Figure 2 shows that the resting tone values tend to decrease (initially $0.122 \mathrm{mV}$ - finally $0.90 \mathrm{mV}$ ). We assume that there is a connection between the decrease of the resting tone and the increase of the muscle contraction capacity, but this is not constant, and the results cannot be generalised.

Table 3. Muscle contraction values ( $m V$ ) for the subject P.G.

\begin{tabular}{lcccc}
\hline \multicolumn{1}{c}{ Measured muscle } & MIVR $(\mathrm{mV})$ & FMVR $(\mathrm{mV})$ & IVMC $(\mathrm{mV})$ & FVMC $(\mathrm{mV})$ \\
\hline R. pectoralis major & 0.130 & 0.012 & 0.746 & 2.344 \\
L. pectoralis major & 0.092 & 0.028 & 0.672 & 1.932 \\
R. rectus abdominis & 0.120 & 0.090 & 0.746 & 0.576 \\
L. rectus abdominis & 0.094 & 0.142 & 0.672 & 0.674 \\
R. quadriceps & 0.071 & 0.015 & 3.361 & 4.026 \\
L. quadriceps & 0.050 & 0.036 & 2.189 & 1.360 \\
R. tibialis anterior & 0.119 & 0.026 & 1.665 & 1.805 \\
L. tibialis anterior & 0.035 & 0.023 & 1.711 & 2.385 \\
R. rhomboid major & 0.090 & 0.106 & 1.273 & 1.186 \\
L. rhomboid major & 0.047 & 0.041 & 2.004 & 1.802 \\
R. paravertebral & 0.056 & 0.082 & 0.566 & 0.809 \\
L. paravertebral & 0.048 & 0.073 & 0.795 & 1004 \\
R. hamstrings & 0.048 & 0.049 & 0.568 & 2.103 \\
L. hamstrings & 0.035 & 0.049 & 1.650 & 2.025 \\
R. triceps surae & 0.048 & 0.033 & 1.744 & 4.195 \\
L. triceps surae & 0.035 & 0.038 & 2.042 & 1.776 \\
\hline Legend: MIVR = initial maximum value at rest, FMVR = final maximum value at rest, IVMC $=$ initial value at maximum contraction,
\end{tabular}

Legend: $M I V R=$ initial maximum value at rest, FMVR = final maximum value at rest, IVMC = initial value at maximum contraction, $F V M C=$ final value at maximum contraction, $R=$ right, $L=$ left, $m V=$ millivolt

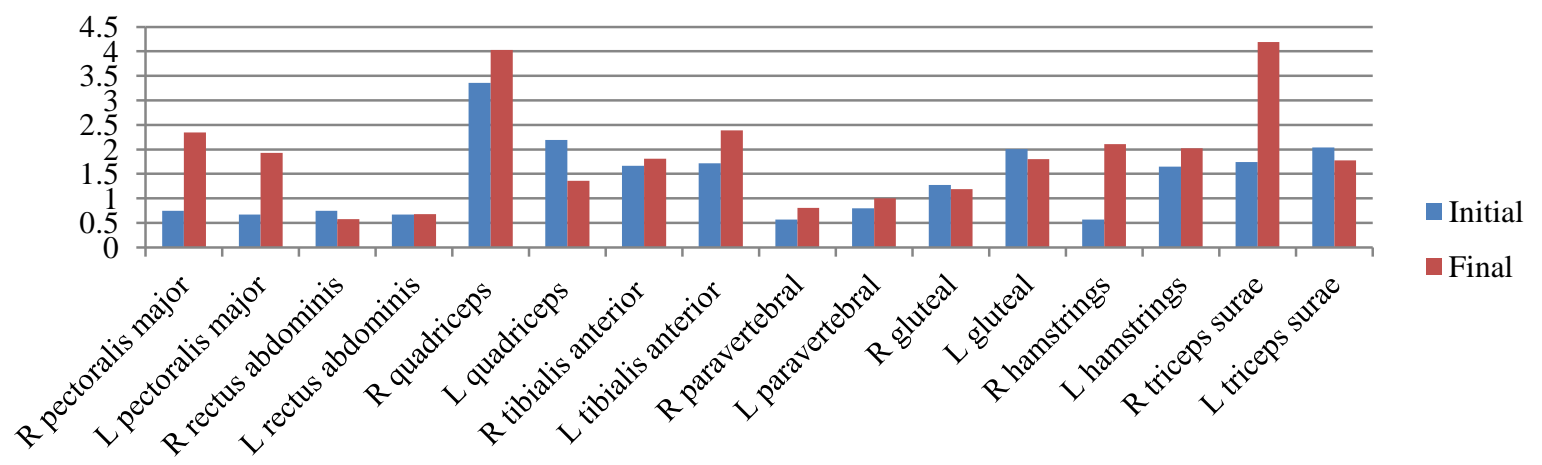

Figure 3. Dynamics of the muscle tone evolution during maximum contraction for the subject P.G.

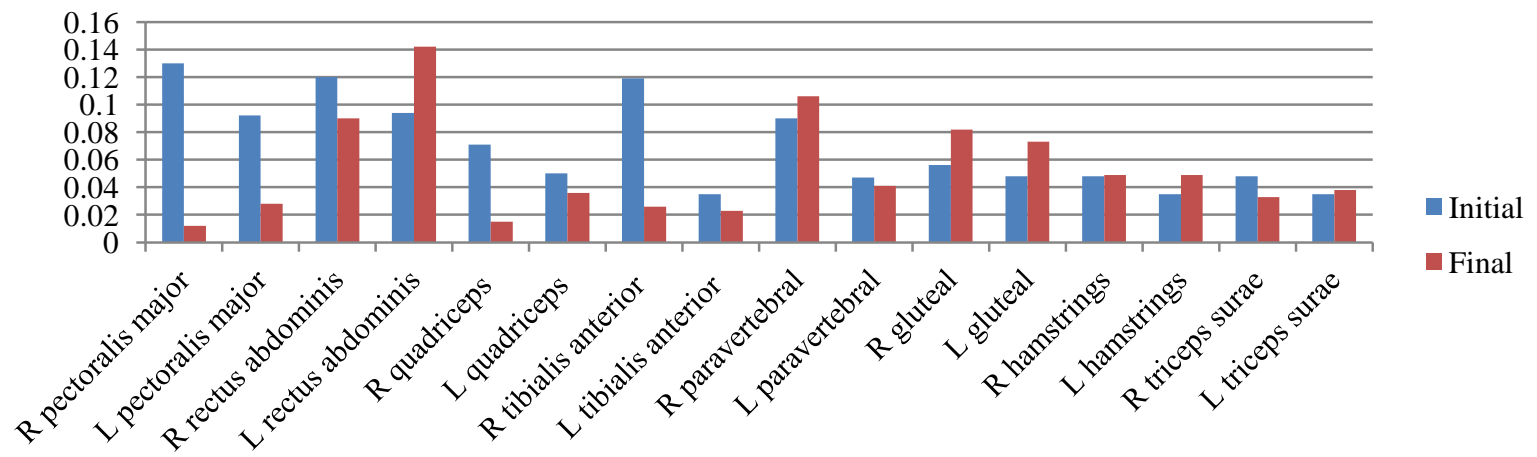

Figure 4. Dynamics of the muscle tone evolution at rest for the subject P.G. 
Figure 3 shows the muscle tone evolution during isometric contraction for the subject P.G. Since the subject had a left flat foot, in the initial evaluation we can observe higher muscle values of the left lower limb, left tibialis anterior muscle $(1.711 \mathrm{mV})$ and left triceps surae muscle $(2.024 \mathrm{mV})$ compared to the right lower limb, right tibialis anterior muscle (1.665 $\mathrm{mV})$ and right triceps surae $(1.744 \mathrm{mV})$. We assume that the flat foot was responsible for the hyper-programming of the right posterior spiral chain, which led to the occurrence of the iliotibial friction syndrome.

In the final evaluation, we can observe that the tone has doubled in the right triceps surae muscle (1.744 $\mathrm{mV}$ initially $-4.195 \mathrm{mV}$ finally) and the activity has increased in the right tibialis anterior muscle ( $1.665 \mathrm{mV}$ initially $-1.805 \mathrm{mV}$ finally). We believe that the doubling of the triceps surae muscle values has led to increased activity in the left posterior spiral chain, helping to rebalance the posterior muscle chains $(1.220 \mathrm{mV}$ initial left posterior spiral chain value - $2.227 \mathrm{mV}$ final left posterior spiral chain value).

The resting tone, highlighted in Figure 4, had a downward trend. Out of the 16 initially evaluated muscles, 12 had lower resting tone values. The same trend is also for the subject no. 1 - lowering the resting tone value leads to an increase in contraction capacity. Muscles with contraction values greater than $2.000 \mathrm{mV}$ have a resting tone below $50 \mathrm{mV}$, the connection between resting tone and contraction capacity being confirmed.

Table 4. Muscle contraction values $(\mathrm{mV})$ for the subject $S . O$.

\begin{tabular}{lcccc}
\hline \multicolumn{1}{c}{ Measured muscle } & MIVR $(\mathrm{mV})$ & FMVR $(\mathrm{mV})$ & IVMC $(\mathrm{mV})$ & FVMC $(\mathrm{mV})$ \\
\hline R. pectoralis major & 0.029 & 0.105 & 0.857 & 0.415 \\
L. pectoralis major & 0.104 & 0.043 & 1.035 & 0.423 \\
R. rectus abdominis & 0.139 & 0.144 & 1.106 & 0.886 \\
L. rectus abdominis & 0.088 & 0.092 & 0.781 & 0.405 \\
R. quadriceps & 0.040 & 0.032 & 0.880 & 1.684 \\
L. quadriceps & 0.052 & 0.012 & 1.683 & 2.344 \\
R. tibialis anterior & 0.052 & 0.072 & 1.675 & 1.917 \\
L. tibialis anterior & 0.059 & 0.040 & 2.820 & 2.516 \\
R. rhomboid major & 0.019 & 0.058 & 1.721 & 1.347 \\
L. rhomboid major & 0.030 & 0.045 & 1.970 & 2.118 \\
R. paravertebral & 0.060 & 0.074 & 1.684 & 2.029 \\
L. paravertebral & 0.037 & 0.077 & 1.600 & 1.247 \\
R. hamstrings & 0.078 & 0.045 & 1.242 & 0.410 \\
L. hamstrings & 0.079 & 0.035 & 1.453 & 0.529 \\
R. triceps surae & 0.022 & 0.088 & 1.008 & 1.665 \\
L. triceps surae & 0.009 & 0.140 & 0.603 & 1.299 \\
\hline L & . & & &
\end{tabular}

Legend: $M I V R=$ initial maximum value at rest, FMVR = final maximum value at rest, IVMC $=$ initial value at maximum contraction, $F V M C=$ final value at maximum contraction, $R=$ right, $L=$ left, $m V=$ millivolt 


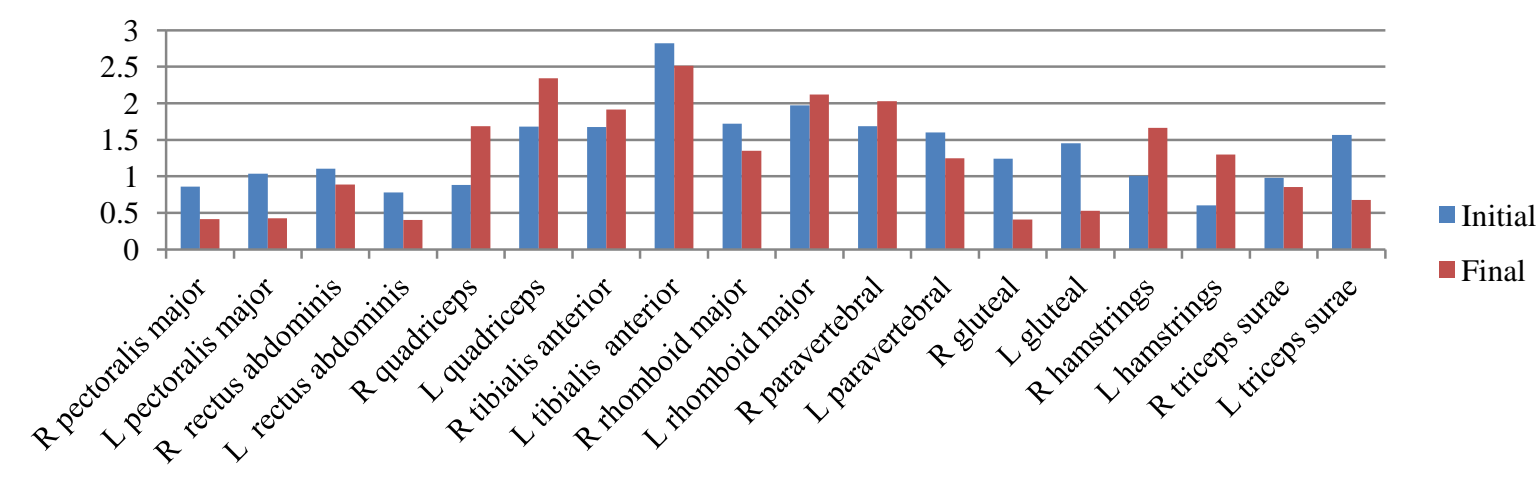

Figure 5. Dynamics of the muscle tone evolution during maximum contraction for the subject S.O.

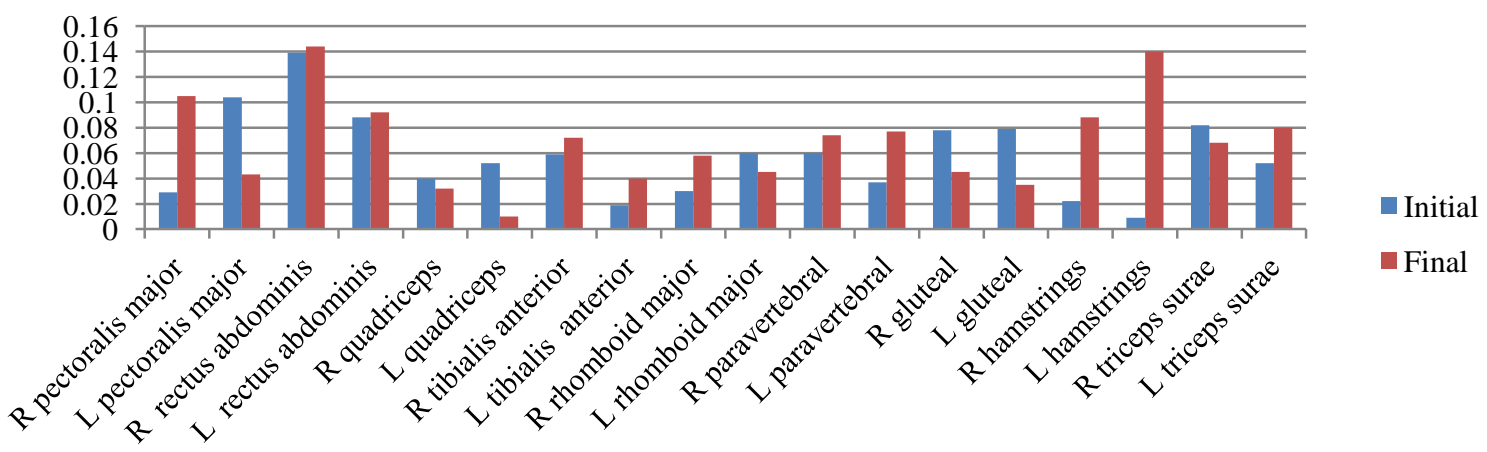

Figure 6. Dynamics of the muscle tone evolution at rest for the subject S.O.

Due to a pre-existing femoral injury, the muscle tone values highlighted in Figure 5 were not interpreted according to the muscle chains.

By performing an individual analysis of the right lower limb muscles, we can notice that the quadriceps muscles (initially: 0.880 right - 1.683 left, finally: 1.684 right -2.344 left), tibialis anterior muscles (initially: 1.675 right -2.820 left, intermediate: 1.917 right -2.516 left) and triceps surae muscles (initially: 0.979 right - 1.568 left, intermediate: 0.851 right 0.677 left) have half the values of the counter side limb. These values are lower due to the presence of pain during running, the subject avoiding the load on the right leg and thus the muscles losing their tone. In the final evaluation, when the subject resumed training, these values became closer, the differences between them being almost inexistent.

Another important aspect is that, during the $4^{\text {th }}$ week of treatment, the subject underwent a second-degree muscle strain as a result of inappropriate warm-up. From the 4th week of treatment until the final evaluation, the intensity of the training was diminished, the athlete being integrated into a recovery programme prescribed by the physical therapist. Although the athlete should have been $100 \%$ recovered at the final evaluation, the disregard of the resting period and muscle overload during training led to recurrence.

By analytically evaluating the muscle contraction values, we can see that the strain of the left hamstring muscle has reduced the contraction capacity of the entire muscle chain, the results being more evident on the muscles with which it had functional connections - left 
triceps surae (initially $1.568 \mathrm{mV}-0.677 \mathrm{mV}$ finally) and gluteal muscles (initially $1.242 \mathrm{mV}$ - $0.410 \mathrm{mV}$ finally).

The resting tone values shown in Figure 6 indicate an average increase of $0.012 \mathrm{mV} /$ muscle from the initial value, probably due to the existing injury.

Table 5. Muscle contraction values ( $m V$ ) for the subject U.S.

\begin{tabular}{|c|c|c|c|c|}
\hline Measured muscle & $\operatorname{MIVR}(\mathrm{mV})$ & FMVR (mV) & IVMC $(\mathrm{mV})$ & FVMC (mV) \\
\hline R. pectoralis major & 0.151 & 0.023 & 1.936 & 1.613 \\
\hline L. pectoralis major & 0.212 & 0.062 & 0.538 & 0.856 \\
\hline R. rectus abdominis & 0.120 & 0.086 & 1.411 & 1.057 \\
\hline L. rectus abdominis & 0.016 & 0.082 & 2.737 & 3.605 \\
\hline R. quadriceps & 0.035 & 0.119 & 1.636 & 1.997 \\
\hline L. quadriceps & 0.052 & 0.090 & 2.859 & 2.767 \\
\hline R. tibialis anterior & 0.022 & 0.032 & 3.829 & 2.738 \\
\hline L. tibialis anterior & 0.042 & 0.050 & 1.526 & 2.557 \\
\hline R. rhomboid major & 0.052 & 0.045 & 1.655 & 2.268 \\
\hline L. rhomboid major & 0.048 & 0.110 & 1.128 & 1.356 \\
\hline R. paravertebral & 0.021 & 0.036 & 1.087 & 0.865 \\
\hline L. paravertebral & 0.075 & 0.012 & 1.120 & 1.638 \\
\hline R. hamstrings & 0.094 & 0.061 & 1.443 & 1.185 \\
\hline L. hamstrings & 0.017 & 0.033 & 2.415 & 1.273 \\
\hline R. triceps surae & 0.063 & 0.008 & 1.410 & 1.743 \\
\hline L. triceps surae & 0.026 & 0.040 & 0.655 & 1.190 \\
\hline
\end{tabular}

Legend: $M I V R=$ initial maximum value at rest, FMVR $=$ final maximum value at rest, IVMC $=$ initial value at maximum contraction, $F V M C=$ final value at maximum contraction, $R=$ right, $L=$ left, $m V=$ millivolt

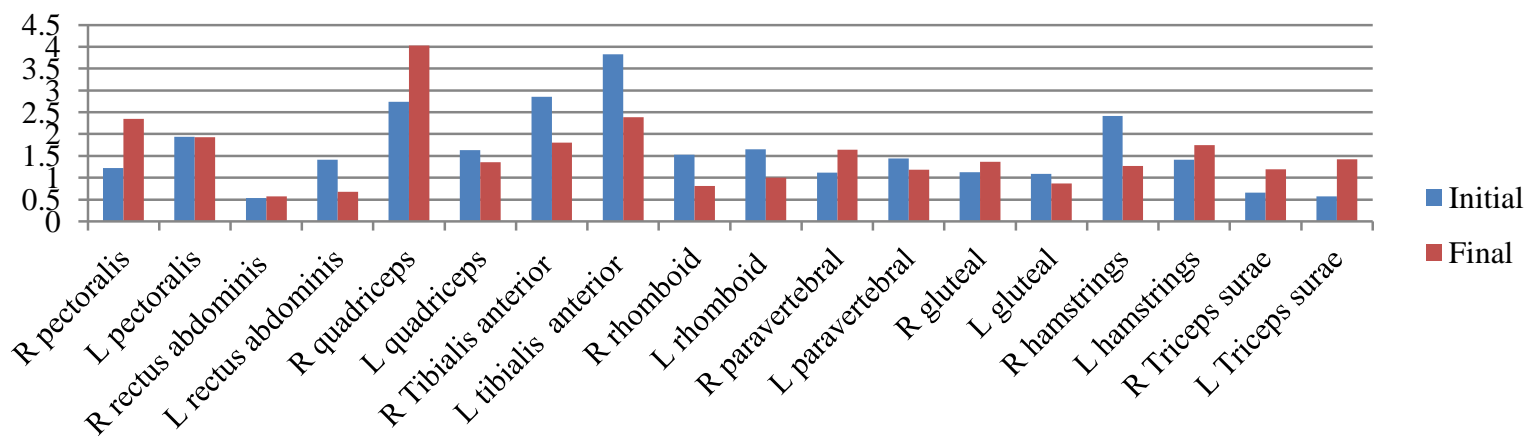

Figure 7. Dynamics of the muscle tone evolution during maximum contraction for the subject S.U.

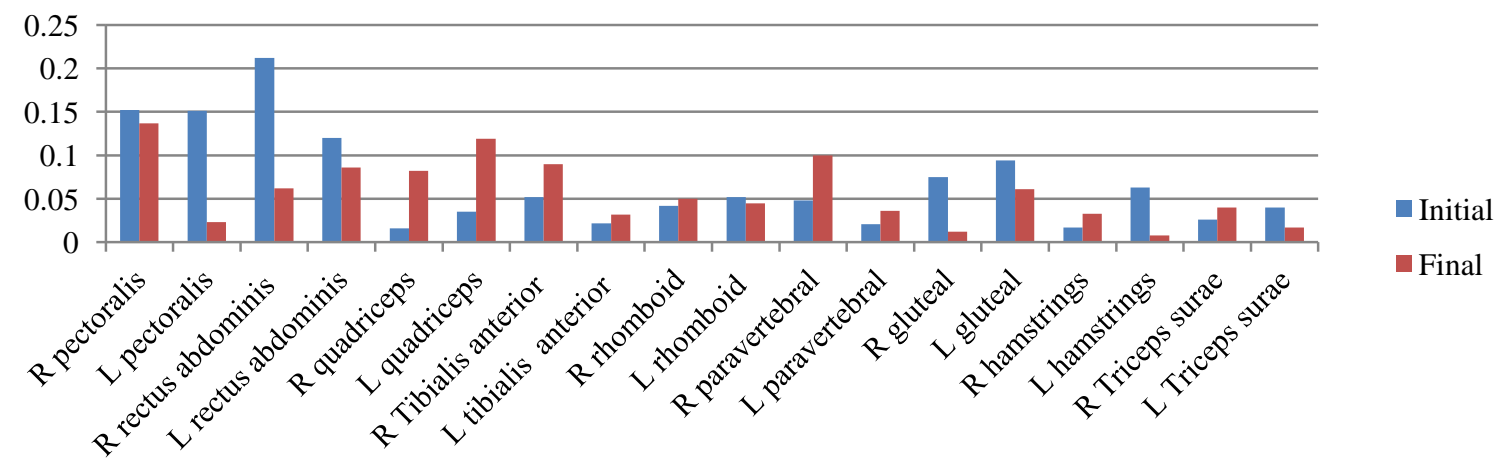

Figure 8. Dynamics of the muscle tone evolution at rest for the subject S.U. 
Figure 7 shows the evolution of maximum contraction values for the subject S.U. There are no important differences between the initial values and the final values, the changes only occur when assessing the muscle chains. In the initial assessment, the functional flexion chain was dominant $(1.958 \mathrm{mV} /$ contraction), resulting in a final estimate of $0.171 \mathrm{mV}(1.787$ $\mathrm{mV} /$ contraction). The functional extension chain had an initial average value of (1.365 $\mathrm{mV} /$ contraction), and at the end, it had a value of (1.807 $\mathrm{mV} /$ contraction), the difference being $0.451 \mathrm{mV}$. The subject S.U. had the greatest increase of the functional extension chain ( $0.450 \mathrm{mV} /$ contraction). This increase is normal because the athlete has a kyphotic attitude. Together with the release of the anterior trunk muscles (pectoralis, rectus abdominis), the posture improved allowing a better extension of the chest, therefore a stronger contraction of the spiral chain. Once the functional chain expansion values grew, we can see an increase of $0.200 \mathrm{mV} /$ contraction in the anatomical extension chain.

Figure 8 shows the resting tone evolution, but, because it presents fluctuations, the results cannot be correlated with the data in the literature, or with the data encountered in other athletes.

\section{Discussion}

Due to the ease with which myofascial release techniques can be applied, they have become a very important tool for preventing the occurrence of musculoskeletal disorders, but also for medical recovery. In terms of their importance in performance sport, they have proven to be effective in the treatment of strains and muscle tears (Ekta et al., 2013), sprains and joint injuries (Cashman et al., 2014), as well as in the lumbar or cervical spine pain (Arguisuelas et al., 2017).

Kalichman and Ben David (2017) conducted a literature review, which included the largest databases in the world (PubMed, Google, Scholar, PEDro), to summarise the effects of myofascial release techniques. The authors concluded that they have biomechanical effects (by increasing the joint range of motion, without reducing the muscle contraction capacity), as well as physiological (by stimulating the blood circulation), neurological (through the pressures exerted on the proprioceptors existing in the fascial tissue) and psychological effects (by inducing a state of relaxation).

Identical results were also found by MacDonald et al. (2012), who applied a foam rolling protocol (a new myofascial release technique) on 11 subjects and did not record a decrease in muscle strength 2 and 10 minutes after applying the foam rolling myofascial release technique.

Arroyo-Morales et al. (2008) suggest the possibility of a transient loss of muscle strength caused by the modification of the tension-length ratio of the muscle fibre. A change in muscle fibre length with a transient loss of muscle strength may be related to a change in muscle architecture, but this change does not affect the values of long-term muscle contraction.

Other research suggests the importance of myofascial release techniques in: preventing the occurrence of injuries, improving local and global blood circulation, decreasing muscle soreness, diminishing inflammatory processes, stimulating the sympathetic nervous system function (an aspect that can facilitate the recovery of athletes), stimulating the immune 
system and improving sports performance (Beardsley \& Škarabot, 2015; Cheatham et al., 2015).

The results obtained in our research are confirmed by the existing publications in the literature. In our research, we obtained a decrease in resting muscle tone and an improvement in isometric contraction capacity. These results are supported by the physiological effects of the myofascial release techniques mentioned above. Research on myofascial chains is at the beginning, but if it proves to be clinically efficient, we will be able to have a more comprehensive approach to the patient.

Summarising all the aforementioned research, in order to avoid any negative effects of myofascial release techniques on sports performance, we recommend that they should not be applied prior to training or competition. Although research does not indicate a decrease in muscle activity, the induced relaxation state may adversely affect the performance of athletes.

\section{Conclusion}

The intervention by using myofascial techniques based on the muscle chain concept leads to the rebalancing of muscle tone. We emphasise that:

- as a result of the treatments applied, we have succeeded in reducing the resting tone in all athletes. Together with the decrease of the resting tone, there was also a tendency to increasing maximum isometric contraction;

- all participants experienced increases in their muscle activity on the functional extension chain, especially those practising short-distance events (400-1,500 meters), as a result of the athlete's typology and the type of exercise practised in accordance with the running biomechanics;

- the central nervous system dictates the functional motion patterns in relation to the peripheral tension and according to the motor engrams formed by the repetitive movements specific to the sports event;

- the best results were obtained by the athletes who followed the physical therapist's instructions, except for S.O., who did not follow the treatment protocol, which led to the prolongation of inactivity. Apart from the subject S.O., no athlete suffered injuries during the treatment period;

- the application of myofascial release techniques according to a well-established clinical judgment can be very effective in the treatment of any musculoskeletal pathology;

- the coach-athlete-physical therapist collaboration is the success of an effective strategy to prevent injuries and increase sports performance.

\section{References}

Ajimsha, M., Al-Mudahka, N. R., \& Al-Madzhar, J. (2015). Effectiveness of myofascial release: Systematic review of randomized controlled trials. Journal of Bodywork and Movement Therapies, 19(1), 102-112. https://doi.org/10.1016/j.jbmt.2014.06.001

Arguisuelas, M. D., Lisón, J. F., Sánchez-Zuriaga, D., Martínez-Hurtado, I., \& DoménechFernández, J. (2017). Effects of myofascial release in nonspecific chronic low back pain. SPINE, 42(9), 627-634. https://doi.org/10.1097/brs.0000000000001897 
Arroyo-Morales, M., Olea, N., Martínez, M. M., Hidalgo-Lozano, A., Ruiz-Rodríguez, C., \& Díaz-Rodríguez, L. (2008). Psychophysiological effects of massage-myofascial release after exercise: A randomized sham-control study. The Journal of Alternative and Complementary Medicine, 14(10), 1223-1229. https://doi.org/10.1089/acm.2008.0253

Beardsley, C., \& Škarabot, J. (2015). Effects of self-myofascial release: A systematic review. Journal of Bodywork and Movement Therapies, 19(4), 747-758. https://doi.org/10.1016/j.jbmt.2015.08.007

Budiman, M. (2009). Understanding the process of fascial unwinding. International Journal of Therapeutic Massage and Bodywork. 2(3), 10-17. https://dx.doi.org/10.3822\%2Fijtmb.v2i3.43

Cashman, G. E., Mortenson, W. B., \& Gilbart, M. K. (2014). Myofascial treatment for patients with acetabular labral tears: A single-subject research design study. Journal of Orthopaedic \& Sports Physical Therapy, 44(8), 604-614. https://doi.org/10.2519/jospt.2014.5095

Cheatham, S. W., Kolber, M. J., Cain, M., \& Lee, M. (2015). The effects of self-myofascial release using a foam roll or roller massager on joint range of motion, muscle recovery, and performance: A systematic review. International Journal of Sports Physical Therapy, 10(6), 827-838. https://pubmed.ncbi.nlm.nih.gov/26618062/

Dischiavi, S. L., Wright, A. A., Hegedus, E. J., \& Bleakley, C. M. (2018). Biotensegrity and myofascial chains: A global approach to an integrated kinetic chain. Medical Hypotheses, 110, 90-96. https://doi.org/10.1016/j.mehy.2017.11.008

do Rosário, J. L. P., Nakashima, I. Y., Rizopoulos, K., Kostopoulos, D., \& Pasqual Marques, A. (2012). Improving posture: Comparing segmental stretch and muscular chains therapy. Clinical Chiropractic, 15(3-4), 121-128. https://doi.org/10.1016/j.clch.2012.10.039

Ekta, C., Nehal, S., Neeta, V., Ratan, K., Dhara, C., \& Gopal, N. (2013). Comparative study of myofascial release and cold pack in upper trapezius spasm. International Journal of Health Science and Research, 3, 20-27. doi: 10.7860/JCDR/2018/37558.12218

Huijing, P. A. (2009). Epimuscular myofascial force transmission: A historical review and implications for new research. International Society of Biomechanics, 42(1), 9-21. https://doi.org/10.1016/j.jbiomech.2008.09.027

Huijing, P. A. (2012). Myofascial force transmission. In F. C. Moore (Ed.), Encyclopedia of exercise medicine in health and disease. Berlin: Springer.

Kalichman, L., \& Ben David, C. (2017). Effect of self-myofascial release on myofascial pain, muscle flexibility, and strength: A narrative review. Journal of Bodywork and Movement Therapies, 21(2), 446-451. https://doi.org/10.1016/j.jbmt.2016.11.006

Krause, F., Vogt, L., \& Banzer, W. (2016). What is evidence-based about myofascial chains. Archives of Physical Medicine and Rehabilitation, 97(3), 454-461. https://doi.org/10.1016/j.apmr.2015.07.023

Lee, S., Joo, K. B., \& Song, S.-Y. (2011). Accurate definition of superficial and deep fascia. Radiology, 261(3), 994-994. https://doi.org/10.1148/radiol.11111116

MacDonald, G. Z., Penney, M. D. H., Mullaley, M. E., Cuconato, A. L., Drake, C. D. J., Behm, D. G., \& Button, D. C. (2013). An acute bout of self-myofascial release increases range of motion without a subsequent decrease in muscle activation or force. Journal of Strength and Conditioning Research, 27(3), 812-821.

https://doi.org/10.1519/jsc.0b013e31825c2bc1

Maganaris, C. N., \& Paul, J. P. (2000). Hysteresis in intact human tendon. Journal of Biomechanics, 33(12), 1723-1727. https://doi.org/10.1016/s0021-9290(00)00130-5

Manheim, C. (2001). The myofascial release manual $\left(3^{\text {rd }}\right.$ ed.). New Jersey: Slack Incorporated. 
Myers, T. W. (2009). Anatomy trains: Myofascial meridians for manual and movement therapists $\left(2^{\text {nd }}\right.$ ed.). Toronto: Churchill Livingstone.

Richter, P., \& Hebgen, E. (2009). Trigger points and muscle chains in osteopathy. New York: Thieme.

Stecco, L. (2004). Fascial manipulation for musculoskeletal pain. Padova: Piccin Nuova.

Stecco, M. C., \& Day, P. J. A. (2010). The fascial manipulation technique and its biomedical model: A guide to the human fascial system. International Journal of Therapeutic Massage \& Bodywork: Research, Education, \& Practice, 3(1), 38-40. https://doi.org/10.3822/ijtmb.v3i1.78

Tozzi, P. (2012). Selected fascial aspects of osteopathic practice. Journal of Bodywork \& Movement Therapies, 16(4), 503-519. https://doi.org/10.1016/j.jbmt.2012.02.003 